\section{Quantum dissipation and Neural Net Dynamics}

\author{
Eliano Pessa ${ }^{a}$ and Giuseppe Vitiello ${ }^{b}$ \\ ${ }^{a}$ Facoltà di Psicologia, Università di Roma \\ "Sapienza" \\ Via dei Marsi 78, 00185 Roma, Italia \\ and ECONA, Interuniversity Center for \\ Research \\ on Cognitive Processing in Natural and \\ Artificial Systems \\ Roma, Italia \\ ${ }^{b}$ Dipartimento di Fisica, Università di Salerno \\ 84100 Salerno, Italia \\ and INFM Unità di Salerno \\ pessa@axcasp.caspur.it \\ vitiello@physics.unisa.it
}

\begin{abstract}
Inspired by the dissipative quantum model of brain, we model the states of neural nets in terms of collective modes by the help of the formalism of Quantum Field Theory. We exhibit an explicit neural net model which allows to memorize a sequence of several informations without reciprocal destructive interference, namely we solve the overprinting problem in such a way last registered information does not destroy the ones previously registered. Moreover, the net is able to recall not only the last registered information in the sequence, but also anyone of those previously registered.
\end{abstract}

The quantum model of brain by Umezawa and Ricciardi [1, 2, 3, 4] has attracted much attention in recent years. Moreover, its extension to dissipative dynamics [5], aimed to solve the long standing problem of memory capacity, provides an interesting framework to study consciousness related mechanisms. On the other hand, computational neuroscience mostly relies on specific activity of neural cells and of their networks, thus leading to a number of models and simulations of the brain activity in terms of neural nets, mostly based on modern methods of statistical mechanics and of spin glass theory [6, 7]. Besides, there is an increasing interest in the study of quantum features of network dynamics, either in connection with information processing in biological systems, or in relation with a computational strategy based on the system quantum evolution (quantum computation).

Inspired thus by the papers [1, 2, 3] and [5] (see also [8, 9, 10]), we explore the possibility of modeling the states of neural nets in terms of collective modes by the help of the formalism of Quantum Field Theory (QFT).

We show that the classical limit of the dissipative quantum brain dynamics (DQBD) [5] provides a representation of a neural net characterized by long range correlations among the net's units. In this way we exhibit a link between DQBD and neural net dynamics [11].

We present an explicit neural net model which allows to memorize a sequence of several informations without reciprocal destructive interference, namely we solve the overprinting problem, i.e. last registered information does not destroy the ones previously registered. The net is also able to recall informations registered prior to the last registered one in the sequence.

In the following we will first introduce the general theoretical background on which our neural net is modeled and then we will present some of its specific features and the results, which, although preliminary, confirm our expectations. 
We consider a three-dimensional set of $N$ interacting units (neural units) sitting each one in a space-time site $x_{n} \equiv\left(\mathbf{x}_{n}, t_{n}\right), n=$ $1,2, . . N$. Each unit can be in the state on (1) or off $(0)$. The neural unit activity is characterized by the amplitude of the emitted pulse and by the phase determined by the emission time. This suggests to us that each unit can be described by a complex doublet field $\psi\left(x_{n}\right)=\left(\psi_{u}\left(x_{n}\right), \psi_{d}\left(x_{n}\right)\right)$, with $\psi_{u}\left(x_{n}\right)$ and $\psi_{d}\left(x_{n}\right)$ complex field components, $u$ and $d$ denoting the field inner degrees of freedom corresponding to on and of $f$, respectively.

At each site $x_{n}$ the field inner variable may assume a well specified value ( $u$ or $d)$. The set of these values for all the sites specifies the microscopic configuration in the $(u-d)$ space; however, in full generality the specification of the macroscopic or functional state of the net does not actually requires that correspondingly one should have a unique, well definite microscopic configuration where each unit state is specified by a definite $u$ or $d$ value. In general, indeed, many distinct microscopic configurations of the component units may correspond to the same functional state of the net.

This means that a given (equilibrium) state of the net may be well compatible with fluctuations in the states of the individual component units. This amounts to say that the net state is not strictly and crucially dependent on the specific state of each individual unit: i.e. we admit enough plasticity (as contrasted with rigidity) for the net; in other words, we can say that the net macroscopic state is the output, or the asymptotic state, emerging from the microscopic dynamics which rules the interaction among the component units.

For large number $N$ of component units, such a picture is certainly more "realistic" than a "rigid" one and could also be more appropriate for a possible modeling of the natural brain in terms of neural nets (as it is well known the brain functional activity is not strictly related with the activity of each single neuron; in this paper however we will not deal with modeling the natural brain).

One can view such a situation also from the perspective of the pulses or signals traveling on the connections among the units: a traveling signal may contribute to excite or de-excite a certain unit thus changing its $u$ or $d$ state. Consequently, a specific state of the net, corresponding to a given dynamical distribution of pulses on the net connections, is necessarily a state for which the single unit states at each site cannot be uniquely specified once for ever, due to pulse action on the units. As a matter of fact, one should consider the unit states as non-observable, since any observation on the unit may nontrivially interfere with the dynamics of the pulses. Only the output of such a dynamics is observable and this is why above we have called it the asymptotic state of the net (i.e. states for time $t_{n} \rightarrow \pm \infty$ for each $n$ ).

Summing up, since fluctuations are allowed for the states of the individual unit at each site, and thus for the basic field $\psi\left(x_{n}\right)$, and since, as a consequence, in the $(u-d)$ space the uncertainty in the identification of the "trajectory" representing the evolution of the state of the unit cannot be eliminated without strongly interfering with it, we are led to treat $\psi\left(x_{n}\right)$ like a quantum field satisfying quantum dynamical equations.

The above considerations lead us to think of the neural net in terms similar to the ones usually adopted for condensed matter physics: the global behavior of the net, namely its functional state and its evolution, can be characterized by a (classical) macroscopic observable as it usually happens in solid state physics, e.g. in superconductivity, in ferromagnetism, etc.. Such an observable, generally called the "order parameter", is de- 
termined by the dynamics of the elementary components or units and by its symmetries [12, 13, 14, 15]. It may be considered as a code specifying the vacuum or ground state 回, 每.

Like in ferromagnetism one introduces the order parameter "magnetization", in our present case we introduce the macroscopic observable $\mathcal{M}$ whose values are assumed to specify the information content of the net. We define $\mathcal{M} \equiv(1 / 2)\left|\left(N_{u}-N_{d}\right)\right|$, with $N=$ $N_{u}+N_{d}$, where $N_{u}$ and $N_{d}$ denote the number of units on and off, respectively. $\mathcal{M}$ is the neural net order parameter which characterizes its macroscopic state.

The state $\mathcal{M}=0$ is called the "normal state" (void of information content); the "information states" or "memory states" are the ones with $\mathcal{M} \neq 0$ (different informations associated to different non-zero $\mathcal{M}$ values).

Since the information comes to the net from the outside, we assume that the neural net may be set into a $\mathcal{M} \neq 0$ state under the action of an external input (coupling of the net with the environment) and it remains in such a state even after the external input is not anymore acting on the net (the information has been recorded). In other words, we assume that the interaction among the net units cannot force, by itself, the net into a $\mathcal{M} \neq 0$ state (i.e. prior of any external input the net remains in its normal state). This in turn means that, from one side, the basic dynamics describing the interaction among the units (i.e. the evolution equations for the basic $\psi\left(x_{n}\right)$ field) must be invariant under the $S U(2)$ group of transformations acting on the doublet field $\psi\left(x_{n}\right)$. On the other side, it also means that the ground state is not invariant under the full $S U(2)$ group of transformations, i.e. the external input triggers the spontaneous breakdown of the $S U(2)$ symmetry.

Notice that, as observed above, there can be many configurations of the set of units (microscopic configurations) corresponding to a given value of $\mathcal{M}$, and therefore to a given state of the net: $\mathcal{M}$ specifies indeed only the difference $\left|\left(N_{u}-N_{d}\right)\right|$, but says nothing on which ones and how many are the sites $u$ and which ones and how many are those $d$; so that any change, or fluctuation, between the $u$ and $d$ state of the units in different sites is allowed, provided the difference $\mathcal{M}$ is kept constant. In this sense $\mathcal{M}$ is a macroscopic variable. On the contrary, the $\psi\left(x_{n}\right)$ fields determine the microscopic configurations.

Moreover, the quantum field dynamics generates asymptotic equilibrium states with negligible fluctuations of $\mathcal{M}$. The macroscopic "memory" state of the neural net indexed by $\mathcal{M}$ is then a classical limit state in the sense of QFT, namely the state for which the fluctuations in the number of certain modes is negligible with respect to the number of the same kind of modes condensed in it: in other words, a coherent state [16] with respect to these modes. These condensed modes are long range correlation modes.

We have in conclusion two levels of description: i) The dynamical level and ii) the asymptotic level. At the dynamical level the interaction among the neural units (represented by basic fields $\psi\left(x_{n}\right)$ ) is ruled by a certain set of dynamical equations, which are assumed to be invariant under the $S U(2)$ group; this level is precluded to observations. At the asymptotic level, the $S U(2)$ symmetry is spontaneously broken and the neural net state is characterized by the order parameter $\mathcal{M}$.

The set of asymptotic fields includes the field describing the non-interacting, "free" (at $t_{n} \rightarrow \pm \infty$ for each $n$ ) units, say $\phi\left(x_{n}\right)$, which also is a complex doublet field with $(u-d)$ inner freedom, and other fields which are generated by the dynamics. By resort- 
ing to well known results in QFT 14, 12], indeed, whenever the order parameter $\mathcal{M}$ is different from zero, the dynamics generates excitation fields describing long range correlations among the units, which are therefore collective modes (the Goldstone theorem). These long range modes are massless and thus their condensation in the ground state does not change its energy; it only produces other (ground) states degenerate in the energy, different among themselves for their condensation content. The stability of the memory states is thus insured. The value of the order parameter $\mathcal{M}$ is a measure of the collective mode condensation in the ground state.

Since the net is an open system (coupled with the environment) and the information storage produces by itself the breakdown of the time-reversal symmetry [5], we consider the QFT for dissipative systems and the neural net state can be then recognized [11] to be a finite temperature state of QFT.

The role of dissipation is crucial in solving the overprinting problem, namely the problem of the net memory capacity: in a sequential information recording each information storage would over-impose itself to the previously recorded one, thus deleting it. In the dissipative dynamics, on the contrary, a large memory capacity is possible since each information is recorded in each of the many degenerate ground states, without destructive interference among them [5].

In other words, dissipation implies that the net overall state may be represented as a superposition of infinitely many degenerate ground states, or memory states, each of them labeled by a different code number and each of them independently accessible to information storage. Many information "files" may then coexist thus allowing a huge memory capacity. Non-unitary equivalence among different memory states acts as a pro- tection against overlap or interference among different informations [5].

In realistic neural net, the finiteness of the "volume" (the number of neural units) and possible defect effects may spoil unitary nonequivalence thus leading to information interferences and distortions.

The retrivial of information is described by "reading off" the mirror modes of the same code number of the information to be recalled. These mirror modes are essentially a "replication signal" of the one responsible for memory storage. The replication signal thus acts as a probe by which one "reads" the stored information. The process of information recalling drives the net into the (macroscopic) memory state of code $\mathcal{M}$ corresponding to that specific information to be retrived.

We also observe that the mirror modes may acquire an effective nonzero mass due to the effects of the system finite size. Such an effective mass then introduces a threshold in the energy to supply in order to trigger the "recall" process. This may lead, from one side, to "difficulties" in the information retrivial; on the other side, it may act as a "protection" against unwanted perturbations and cooperate to the neural net memory state stability.

The study of thermodynamic properties shows that the generator of time evolution of the net state is the system entropy. The stationarity of free energy implies the Bose distribution for the collective modes and the Fermi distribution for the neural unit fields. In this way the traditional activation function for neural net units is recovered. For further details see [5] and [11].

Let us now briefly present some of the features of the specific neural net model we have worked out and the related results. We will give here only a qualitative description of the model. See 11] for formal details. 
The net dynamics is given by the PauliDirac equation for a doublet field $\psi$ interacting with an external magnetic field representing the external input. A spatial discretization of this equation on a two dimensional lattice of $20 \times 20$ sites is adopted, so as to transform the original field equation into a system of coupled ordinary differential equations. Besides the interaction with the external magnetic field, the doublet field also interacts with the mean magnetic field which is generated over the net as a response to the external input. We use the BraggWilliams approximation and the mean magnetic field is taken to be proportional to the magnetization induced by the external input with a proportionality constant given by the Weiss constant $\gamma$. The dynamical equation thus presents a nonlinear term in $\psi$ since the magnetization is by itself given in terms of a bilinear form in $\psi$. The Weiss constant $\gamma$ is also related to the mean value over the whole net of the mean values of the connection strength for each site.

The practical implementation of the net was done through the following steps:

a) after explicit representation of the $\psi$ field components $\psi_{u}$ and $\psi_{d}$ in terms of their real and imaginary parts, we obtained the corresponding four equations from the PauliDirac equation.

b) each of the four component field variables was expressed as a product of the site activation function times the connectivity potential of the site itself.

c) we considered the nearest neighbor approximation for the site connections.

d) after a spatio-temporal discretization, we associated to each unit (i.e. to each site) a sigmoidal activation function characterized by a "temperature" parameter.

e) we assumed an independent evolution for each of the four component field variables. f) we used the modulus squared value of the activation function of each unit to determine the time evolution of the activation dynamics of the unit itself.

g) we used simulated annealing in each process of writing and of reading (recalling).

Through the implementation of the above steps we obtained a neural net able to record a sequence of informations without overprinting (i.e. without destruction of previously registered informations in the course of a subsequent registration process) and to able to recall anyone of the registered informations (i.e. not simply the last one) under presentation of an external input similar to the one to be recalled.

Such results make us confident that a novel conceptual and formal scheme in neural net modeling may be introduced which is based on the simulation of a quantum dynamical evolution.

We are glad to aknowledge partial support from MURST and INFM. 


\section{Bibliography}

[1] L.M.Ricciardi and H.Umezawa, Brain physics and manybody problems. Kibernetik 4, 44 (1967)

[2] C.I.J.Stuart, Y.Takahashi and H.Umezawa, On the stability and nonlocal properties of memory. J. Theor. Biol. 71, 605 (1978)

[3] C.I.J. Stuart, Y. Takahashi and H. Umezawa, Mixed system brain dynamics: neural memory as a macroscopic ordered state. Found. Phys. 9, 301 (1979)

[4] S. Sivakami and V. Srinivasan, A model for memory, J. Theor. Biol. 102, 287 (1983)

[5] G. Vitiello, Dissipation and memory capacity in the quantum brain model. Int. J. Mod. Phys. 9973 (1995)

[6] M.Mézard, G.Parisi and M.Virasoro, Spin glass theory and beyond, World Sci., Singapore 1993

[7] D.J.Amit Modeling brain functions, Cambridge University Press, Cambridge 1989

[8] K.H.Pribram, Languages of the brain, Englewood Cliffs, New Jersey, 1971

[9] K.H.Pribram, Brain and perception, Lawrence Erlbaum, New Jersey, 1991

[10] M.Jibu , K.H.Pribram and K.Yasue, From conscious experience to memory storage and retrivial: The role of quantum brain dynamics and boson condensation of evanescent photons. Int. J. Mod. Phys. B10, 1735 (1996)

[11] E.Pessa and G.Vitiello, in preparation

[12] H.Umezawa H.Matsumoto and M.Tachiki , Thermo Field Dynamics and Condensed States, North-Holland, Amsterdam 1982

[13] H.Umezawa, Advanced field theory: $m i-$ cro, macro and thermal concepts, American Institute of Physics, N.Y. 1993

[14] C.Itzykson and J.Zuber , Quantum Field Theory, McGraw- Hill Inc. N.Y. 1980

[15] P.W.Anderson , Basic Notions of Condensed Matter Physics, AddisonWesley, N.Y. 1984

[16] J.R.Klauder and E.C.Sudarshan, Fundamentals of Quantum Optics, Benjamin, New York, 1968 